\title{
Innovación de Base Científica-Tecnológica desde las Universidades de Iberoamérica
}

\author{
Alfonso Cruz Novoa Ph.D*
}

En los últimos años, la acumulación del conocimiento científico-tecnológico ha experimentado un crecimiento dramático, lo que ha generado continuas y relevantes innovaciones. Esto se puede apreciar en el número de patentes que anualmente conceden los países a inventores de todo el mundo. Su incremento ha sido especialmente relevante en las pasadas 3 décadas donde, por ejemplo, Estados Unidos pasó de conceder 60 mil patentes en 1985 a 325 mil el año 2015.

En los países industrializados el conocimiento es el factor más relevante en la producción de nuevos bienes, servicios y procesos; sobrepasando al capital y al trabajo (Arrow, 1962; Lucas, 1988). Lo anterior, ha transformado a las actividades de creación y transferencia del conocimiento en factores esenciales para el desarrollo económico y social de las naciones (OECD, 2010).

En este contexto, las universidades han adquirido un rol fundamental por tratarse de instituciones creadoras de conocimiento. Ellas tienen el potencial de impactar en la sociedad, las industrias y los mercados; transfiriendo sus avances científico-tecnológicos, generando nuevo valor e incrementando la productividad basada en innovación (Jiménez, 2016). Sin embargo, los procesos de creación y transferencia de conocimientos son complejos, no lineales y resultan de una diversidad de interacciones entre los diferentes actores involucrados (Boni \& Emerson, 2005), todo lo cual es difícil de medir.

Disponer de información fidedigna sobre las actividades de investigación, desarrollo, innovación y emprendimiento $(\mathrm{I}+\mathrm{D}+\mathrm{i}+\mathrm{e})$ se ha vuelto indispensable para diseñar políticas universitarias y públicas efectivas. En este contexto, es que en el año 2012 junto a un equipo de representantes de 17 destacadas universidades iberoamericanas (2 brasileñas, 1 colombiana, 1 chilena, 9 españolas, 2 mexicanas, 2 portuguesas), comenzamos un estudio en esta materia. Su objetivo central fue caracterizar el estado y evolución de los principales procesos, recursos y resultados de los sistemas de $\mathrm{I}+\mathrm{D}+\mathrm{i}+\mathrm{e}$ en 17 instituciones de educación superior.

Para ello, elaboramos un modelo de análisis empírico de los sistemas de $\mathrm{I}+\mathrm{D}+\mathrm{i}+\mathrm{e}$ a nivel de universidades con cuatro actividades centrales: 1 ) investigación básica; 2 ) investigación aplicada; 3 ) protección del conocimiento; y 4) transferencia tecnológica (ver Figura 1). Asumimos que dichas actividades - lejos de seguir un proceso secuencial- actúan más bien como un sistema no lineal en donde existe una permanente interacción entre la investigación básica y aplicada. Por otra parte, dependiendo de la naturaleza y tipo de conocimiento generado, varía la forma y oportunidad de su protección y transferencia a la sociedad.

Figura 1. Modelo del sistema de $\mathrm{I}+\mathrm{D}+\mathrm{i}+\mathrm{e}$ universitario utilizado en el análisis empírico

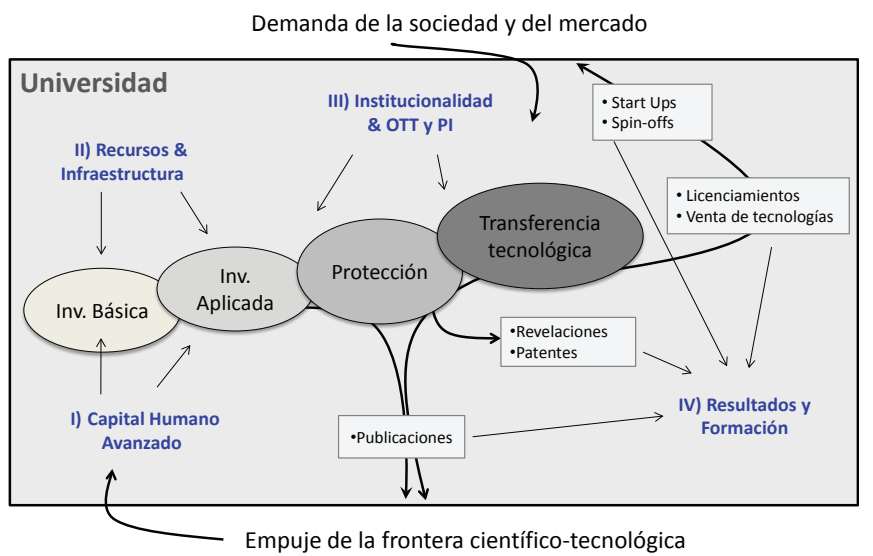

Para que el sistema opere se requiere de un conjunto de insumos, servicios e infraestructura que identificamos a través de cuatro ejes: i) Capital humano avanzado; ii) Recursos e infraestructura; iii) Institucionalidad y servicios; y iv) Formación de capacidades y resultados en $\mathrm{I}+\mathrm{D}+\mathrm{i}+\mathrm{e}$.

En nuestro modelo actúan a su vez dos fuerzas externas relevantes. Por un lado, el "empuje de la frontera científico-tecnológica" para la creación de nuevos conocimientos y, a través de ellos, innovaciones que se transfieren a la sociedad (Dogson, 2000). Por otro lado, la "demanda de la sociedad y del mercado" de innovaciones, mediante nuevas tecnologías, productos y soluciones (Salter \& Martin, 2001). Luego, identificamos 15 variables y 42 indicadores, con los cuales elaboramos una encuesta estructurada que nos permitió caracterizar y medir el sistema de $\mathrm{I}+\mathrm{D}+\mathrm{i}+\mathrm{e}$ en las 17 instituciones iberoamericanas de educación superior entre los años 2007 y 2010.

${ }^{*}$ Profesor de la Escuela de Ingeniería de la Pontificia Universidad Católica de Chile, Director Ejecutivo de la Fundación Copec - UC Email: acruzn@ing.puc.cl 
Entre nuestras conclusiones podemos afirmar que:

Hay avances significativos y sostenidos en el desempeño de los sistemas de $\mathrm{I}+\mathrm{D}+\mathrm{i}+\mathrm{e}$ del conjunto de las instituciones estudiadas

Se observaron mejoras significativas y generalizadas del conjunto de instituciones en las diferentes dimensiones de sus sistemas de creación y transferencia de conocimientos y tecnologías durante el período 2007-2010. Hay un aumento promedio del $20 \%$ en publicaciones y citas WOS, un $60 \%$ en revelaciones de invención y un $28 \%$ en solicitudes de patentes. Hay un incremento de un $114 \%$ en los contratos de licenciamiento por institución y un $25 \%$ en la creación de empresas spin-off.

Todas las universidades estudiadas poseen Oficinas de Transferencia y Licenciamiento (OTL) e incubadoras de empresas. La mayoría dispone de un centro de emprendimiento activo. A su vez, todas tienen o están terminando de institucionalizar procesos orientados a regular la propiedad del conocimiento generado, el licenciamiento de tecnologías y la creación de empresas spin-off.

Lo anterior da cuenta de los avances relevantes y sistemáticos en el conjunto de universidades analizadas y de los esfuerzos que se están desplegando para fomentar la innovación y el emprendimiento de base científico-tecnológico.

En este contexto, las universidades iberoamericanas deben continuar con sus esfuerzos sistemáticos y de largo plazo si quieren asemejarse a las universidades líderes (tanto anglosajonas, europeas como asiáticas). Además, deben apurar el paso en $\mathrm{I}+\mathrm{D}+\mathrm{i}+\mathrm{e}$ y hacer partícipe a la comunidad universitaria (y a la sociedad) de la importancia que tienen este tipo de actividades para el desarrollo de los países. Es clave formar y reclutar el mejor capital humano y dotarse de la infraestructura necesaria para fortalecer la $\mathrm{I}+\mathrm{D}+\mathrm{i}+\mathrm{e}$.

\section{Se observa una gran heterogeneidad en sus políticas y mecanismos de apoyo para la creación y transferencia de conocimiento}

Las 17 universidades estudiadas presentan altos y persistentes niveles de heterogeneidad en sus sistemas de $\mathrm{I}+\mathrm{D}+\mathrm{i}+\mathrm{e}$, los cuales se manifiestan incluso en instituciones de un mismo país con contextos nacionales comunes (por ejemplo, las españolas, mexicanas y brasileñas). Las universidades tienen una alta diversidad de estrategias y políticas de desarrollo de largo plazo, que derivan en distintos comportamientos y desempeños organizacionales.

La alta heterogeneidad se manifiesta en diferencias significativas entre instituciones en los diversos indicadores utilizados en el estudio. Es el caso del gasto en $\mathrm{I}+\mathrm{D}$, el número de alumnos de doctorado, el número de publicaciones indexadas o el número de patentes o contratos de licenciamiento, entre otros. A modo de ejemplo, las universidades estudiadas tuvieron un gasto total directo promedio en I+D de $€ 74$ millones el año 2010, con un presupuesto máximo (€211 millones) que llega a superar en 30 veces al mínimo ( $€ 7$ millones). Además, en promedio, el $21 \%$ de este gasto es financiado con recursos privados externos, con una variación que va desde un máximo del $54 \%$ hasta un mínimo del 7\%.
Una diferencia relevante se puede apreciar en instituciones como el Instituto Tecnológico de Monterrey (ITESM), fuertemente orientado a la formación para el emprendimiento y a la creación de empresas spin-off. El año 2010 el ITESM creó 25 empresas, cifra cuatro veces superior al promedio. Por su parte, la Universidad de Sao Pablo tiene una marcada orientación hacia la investigación básica y a la formación de alumnos de doctorado, que suman más de 13.000 (cuatro veces la media de las universidades analizadas). El alto dinamismo de la Universidad Politécnica de Valencia en materia de patentamiento internacional y transferencia tecnológica es otra singularidad destacable. También el fuerte énfasis de la Universidad de Barcelona por la formación de magíster en innovación y emprendimiento, contando con ocho especialidades diferentes.

La alta y persistente heterogeneidad a nivel de universidades está documentada por la bibliografía y es objeto de interesantes estudios. Su dinámica no se ajusta con lo que ocurre en muchos sectores en donde se observa una tendencia a la convergencia. Esto significa que las diferentes organizaciones de una industria tienden a aumentar su similitud y reducir su heterogeneidad a lo largo del tiempo - fenómeno que en la educación superior no ocurre o sucede en menor grado y más lentamente-. Esta constatación es relevante, ya que nos indica que las instituciones de educación superior tienden a ser persistentemente distintas en sus estrategias de largo plazo.

Como recomendación a lo anterior, debemos distinguir dos niveles de políticas o acciones para tener en cuenta: medidas de carácter general o común a todas las instituciones estudiadas y medidas de carácter local o particular a instituciones específicas. Así, las de carácter general hacen referencia a fomentar buenas prácticas que se pueden incorporar en todas las instituciones con la idea de mejorar los procesos de creación y transferencia de conocimiento. En este sentido, algunos ejemplos de medidas ya implantadas han sido la institucionalización de los reglamentos de propiedad intelectual y transferencia tecnológica - que progresivamente se han ido extendiendo entre las instituciones-. Así como la creación de oficinas de transferencia y licenciamiento (OTL) y la creación de centros de emprendimiento, entre otras.

Por otra parte, las medidas de carácter local, responderán a la orientación estratégica específica de cada institución. Lo anterior implica también, la aplicación de estrategias diferenciadas. En esta línea, los responsables de cada universidad — una vez realizado el diagnóstico de su situación en $\mathrm{I}+\mathrm{D}+\mathrm{i}+\mathrm{e}$ y su benchmarking con respecto a otras instituciones similares y/o de referencia- deberían establecer un plan estratégico con los objetivos que desean alcanzar señalando los aspectos a fortalecer y corregir.

Las universidades iberoamericanas se orientan principalmente a la investigación básica y en menor medida a la aplicada

El conjunto de 17 universidades estudiadas posee un marcado énfasis hacia la investigación básica o fundamental y una menor orientación hacia la investigación aplicada. Por ejemplo, tienen un promedio de 46 solicitudes de patentes por cada 1.000 publicaciones científicas 
indexadas en el período 2007-2010. Sin embargo, esta cifra varía enormemente desde un máximo de 321 solicitudes de patentes por cada 1.000 publicaciones científicas del ITESM y un mínimo de 9 en el caso de las Universidades de Valencia y Autónoma de Madrid.

Como recomendación, es conveniente que las universidades mejoren sus indicadores en investigación aplicada de modo que cubran todo el espectro que va desde la investigación básica pasando por la investigación aplicada y el desarrollo tecnológico. Cuando las universidades tienen bajos niveles de investigación aplicada significa que no están aprovechando las oportunidades que derivan de la investigación básica que desarrollan. Tampoco aprovechan las oportunidades de atraer nuevos recursos - tanto de fuentes públicas como privadas- orientados al financiamiento de la trasferencia tecnológica (TT) y generar así, nuevo valor para la sociedad.

Básicamente se proponen dos ámbitos de acción: 1) Generar y desarrollar ideas potencialmente aplicables; y 2) transferir dichas ideas de modo que logren tener un positivo impacto en la sociedad. Las actuaciones en el primer ámbito buscan incentivar a los investigadores para producir conocimiento y tecnología potencialmente transferibles a la sociedad. Dichas acciones tienen un carácter general y habitualmente conllevan un cambio en la mentalidad de la comunidad académica. Algunas medidas concretas serían la consideración de los esfuerzos y resultados en materia de creación de propiedad intelectual del investigador en los criterios que determinan la promoción en su carrera académica — tal y como ya se viene haciendo en algunos países-. Otra medida posible es el establecimiento de sistemas de incentivos dentro de las universidades que permitan a los académicos percibir un porcentaje de los royalties que se derivan de las patentes o de los contratos de licenciamiento generados.

Por su parte, en lo que se refiere a las actuaciones en el ámbito de la transferencia de ideas, si bien los investigadores desarrollan la tecnología, el personal de la OTL suele tener un mejor conocimiento de su potencial de comercialización. En este sentido, la experiencia de la OTL es fundamental para asesorar a los investigadores en los procesos de protección y transferencia de su invención.

Por otra parte, queremos puntualizar que cuando se recomienda la conveniencia de incrementar el peso de la investigación aplicada no se hace a costa de reducir o restar recursos a la investigación básica. Por el contrario, significa ampliar el espectro de la investigación desde el perfil fundamental hasta el desarrollo de aplicaciones reales. Así, todo el sistema crece y se producen flujos de conocimiento en ambos sentidos.

\section{Las acciones de gestión de la propiedad intelectual (PI) están más avanzadas que las de trasferencia tecnológica}

Dentro del avance sostenido y relevante de las universidades en materia de $\mathrm{I}+\mathrm{D}+\mathrm{i}+\mathrm{e}$, se aprecia que las iniciativas relacionadas con la gestión de la propiedad del conocimiento generado están más avanzadas y desplegadas que las de transferencia tecnológica (que son más recientes y seguramente por ello están menos sistematizadas y son menos intensas).

Así, en el año 2010, el conjunto de 17 instituciones estudiadas solicitó un promedio de 45 patentes de invención (entre nacionales, en el extranjero y vía PCT) y les fueron concedidas un promedio de 22 . A su vez, la media de patentes concedidas en el período 1990-2010 fue de 114 por institución. Las anteriores cifras contrastan con resultados significativamente menores en materia de transferencia de estos conocimientos. En efecto, en el año 2010 las universidades tuvieron un promedio de solo 8 contratos de licenciamiento de tecnologías y los ingresos medios por licenciamiento se situaron en torno a $€ 153.000$ por institución anualmente, es decir muy bajos y esporádicos en el contexto internacional. Con respecto a los ingresos por participación en empresas de tipo spin-off, la situación es aún más incipiente. De hecho, son muy pocas las universidades que reportaron haber obtenido ingresos por participar en este tipo de empresas. Las cifras son también muy pequeñas en materia de ingresos obtenidos por venta de tecnologías.

Como recomendación, se debe avanzar hacia etapas más evolucionadas y sistemáticas de transferencia de los conocimientos generados. Esto pasa por promover relaciones más estrechas con el sector productivo que, en definitiva, es el demandante último de las invenciones desarrolladas en las universidades. Para incrementar dicha colaboración se podrían crear mayores incentivos para la incorporación temporal de doctores a las empresas que tengan capacidades de absorción de nuevo conocimiento. Por su parte, los investigadores debieran de ampliar sus líneas de investigación a las necesidades de nuevo conocimiento y soluciones que la sociedad demanda, así como la celebración de encuentros y jornadas específicas entre investigadores y empresas.

En este estrechamiento de relaciones entre universidad, sector productivo y sociedad en su conjunto, es recomendable que las universidades se abran a incorporar con mayor contundencia un enfoque en la demanda de $\mathrm{I}+\mathrm{D}$ (market pull). Sin duda resulta más fácil transferir una nueva tecnología diseñada para solucionar un problema existente, que crear un nuevo conocimiento tecnológico para después buscar potenciales interesados en adquirirlo (technology push). Esta medida facilitaría la transferencia tecnológica y mejoraría el impacto y los retornos económicos y no económicos de los esfuerzos de la $\mathrm{I}+\mathrm{D}$ en las universidades iberoamericanas.

En resumen, el estudio plasmado en el libro "Análisis de las actividades de investigación + desarrollo + innovación + emprendimiento en universidades de Iberoamérica" (Cruz, 2014); nos ha permitido conocer los avances que las 17 universidades están teniendo en su capacidad para crear y transferir conocimiento pertinente hacia la sociedad. También nos semana, las principales debilidades y carencias que deben ser atendidas para profundizar y consolidar lo avanzado. 


\section{Referencias bibliográficas:}

Arrow, K. (1962). The economic implications of learning by doing. The Review of Economic Studies, 29(3), 155-173.

Boni, A. A., \& Emerson, S. T. (2005). An integrated model of university technology commercialization and entrepreneurship education. University entrepreneurship and technology transfer: Process, design, and intellectual property, 241-274.

Dodgson, M. (2000). Systemic integration of the innovation process within the firm. Department of Industry, Science and Resources.

Cruz, A. (2014). Análisis de las actividades de investigación + desarrollo + innovación + emprendimiento en universidades de Iberoamérica. Colección Estudios Red Emprendia.
Jiménez, A. (2016). Relaciones universidad-empresa: Hacia una productividad basada en innovación. Gestión y Tendencias, 2(1), 7-10.

OECD. (Mayo de 2010). Ministerial report on the OECD Innovation Strategy: Innovation to strengthen growth and address global and social challenges.

Lucas, R. (1988). On the mechanics of economic development. Journal of Monetary Economics, 22(1), 3-42.

Salter, A., \& Martin, B. (2001). The economic benefits of publicly funded basic research: a critical review. Research Policy, 30, 509-524. 